\title{
Stability and Stabilization of a Class of Boundary Control Systems
}

\author{
J.A. Villegas, H. Zwart, Y. Le Gorrec, B. Maschke, and A.J. van der Schaft
}

\begin{abstract}
We study a class of partial differential equations on a one dimensional spatial domain with control and observation at the boundary. For this class of systems we describe how to obtain an impedance energy-preserving system, as well as scattering energy-preserving system. For the first type of systems we consider (static and dynamic) feedback stabilization by means of boundary control. For the scattering energypreserving systems we give conditions for which the system is either asymptotically or exponentially stable.
\end{abstract}

\section{INTRODUCTION}

In this paper we study the following partial differential equation (PDE) on the spatial interval $[a, b]$

$$
\begin{aligned}
\dot{x}(t) & =\mathscr{J} x(t), \quad x(0)=x_{0} \\
u(t) & =\mathscr{B} x(t), \\
y(t) & =\mathscr{C} x(t),
\end{aligned}
$$

here

$$
\mathscr{J} x=\sum_{i=0}^{N} P(i) \frac{d^{i} x}{d z^{i}}(z) \quad z \in[a, b]
$$

with the domain of $\mathscr{J}$ being $H^{N}\left((a, b) ; \mathbb{R}^{n}\right)$, i.e., the Sobolev space of $N$ times differentiable functions on the interval $(a, b) . P(i), i=0, \ldots, N$, is a $n \times n$ real matrix satisfying

$$
P(i)=P(i)^{T}(-1)^{i+1}, \quad \text { and } \quad \operatorname{ker} P(N)=\{0\} .
$$

For the PDE (1) we describe how to obtain impedance energy-preserving systems (systems that satisfy $\left.\frac{1}{2} \frac{d}{d t}\|x(t)\|^{2}=u(t)^{T} y(t)\right)$, as well as scattering energypreserving $\left(\frac{1}{2} \frac{d}{d t}\|x(t)\|^{2}=\|u(t)\|^{2}-\|y(t)\|^{2}\right)$. Next we study feedback (static and dynamic) stabilization for impedance energy-preserving systems. Finally, we study (asymptotic and exponential) stability of scattering energy-preserving.

Here we use the notation $\left[\begin{array}{c}X \\ Y\end{array}\right]$ for $X \times Y$ and $F \mid \mathfrak{D}$ denotes the restriction of an operator $F$ to the subspace $\mathfrak{D} . \rho(F)$ denotes the resolvent set of $F$ and $\partial_{z}^{N}$ indicates the $N$-times partial derivative with respect to the variable $z$. $I$ is the identity operator.

\section{SOME BACKGROUND}

Most of the results described in this section can be found in [1]. First we need to introduce some notation. The $n N \times n N$ matrix $Q$ is defined as

J.A. Villegas, H. Zwart, and A.J. van der Schaft are with Faculty of Electrical Engineering, Mathematics and Computer Science, University of Twente, 7500 AE Enschede, The Netherlands $\{j . a . v i l l e g a s$, h.j.zwart, a.j.vanderschaft $\}$ @math. utwente.nl

Y. Le Gorrec and B. Maschke are with LAGEP, UCB Lyon 1 - UMR CNRS 5007, Bât. 308 G ; 43, Bd du 11 nov. 1918, 69622 Villeurbanne cedex France, $\{$ legorrec, maschke\}@lagep.univ-lyon1.fr

$$
Q=\left(\begin{array}{cccccc}
P(1) & P(2) & P(3) & \cdots & P(N-1) & P(N) \\
-P(2) & -P(3) & -P(4) & \cdots & P(N) & 0 \\
\vdots & \ddots & \ddots & \ddots & & \vdots \\
(-1)^{N-1} P(N) & 0 & \cdots & \cdots & & 0
\end{array}\right)
$$

Using this $Q$, the matrix $R_{\text {ext }}$ is defined as

$$
R_{\mathrm{ext}}=\frac{1}{\sqrt{2}}\left(\begin{array}{cc}
Q & -Q \\
I & I
\end{array}\right) .
$$

Since $P(N)$ is invertible, we have that $R_{\text {ext }}$ is invertible as well. It is easy to see that it satisfies

$$
R_{\mathrm{ext}}^{T} \Sigma R_{\mathrm{ext}}=\left(\begin{array}{cc}
Q & 0 \\
0 & -Q
\end{array}\right) \text {, where } \Sigma=\left(\begin{array}{cc}
0 & I \\
I & 0
\end{array}\right) .
$$

Definition 2.1: The boundary port variables associated with the differential operator $\mathscr{J}$ and the function $x \in$ $H^{N}\left((a, b) ; \mathbb{R}^{n}\right)$ are the vectors $e_{\partial}, f_{\partial} \in \mathbb{R}^{n N}$, defined by

$$
\left(\begin{array}{c}
f_{\partial} \\
e_{\partial}
\end{array}\right)=R_{\text {ext }}\left(\begin{array}{c}
x(b) \\
\vdots \\
\partial_{z}^{N-1} x(b) \\
x(a) \\
\vdots \\
\partial_{z}^{N-1} x(a)
\end{array}\right),
$$

where $R_{\text {ext }}$ is defined by (4).

In [1], the authors prove the following theorem.

Theorem 2.2: Let

$$
W=S[I+V, \quad I-V],
$$

with $S$ invertible and $V V^{T} \leq I$, be a full rank matrix of size $n N \times 2 n N$ (satisfying $W \Sigma W^{T} \geq 0$ ), and define $\mathscr{B}$ : $H^{N}\left((a, b), \mathbb{R}^{n}\right) \rightarrow \mathbb{R}^{n N}$ as

$$
\mathscr{B} x(t):=W\left(\begin{array}{l}
f_{\partial}(t) \\
e_{\partial}(t)
\end{array}\right) .
$$

Then the system (1a)-(1b) is a boundary control system. $A_{W}:=\mathscr{J}_{\mid \operatorname{ker} \mathscr{B}}$ is the generator of a contraction semigroup and

$$
D\left(A_{W}\right)=\left\{x \in L^{2}\left((a, b), \mathbb{R}^{n}\right) \mid\left[\begin{array}{l}
f_{\partial} \\
e_{\partial}
\end{array}\right] \in \operatorname{ker} W\right\} .
$$

Furthermore, if we define the output via the mapping $\mathscr{C}$ : $H^{N}\left((a, b), \mathbb{R}^{n}\right) \rightarrow \mathbb{R}^{n N}$ as

$$
y(t)=\mathscr{C} x(t):=S_{2}\left(\begin{array}{cc}
I-V^{T} & -I-V^{T}
\end{array}\right)\left(\begin{array}{l}
f_{\partial}(t) \\
e_{\partial}(t)
\end{array}\right)
$$

with $S_{2}$ invertible, then for $u \in C^{2}\left((0, \infty) ; \mathbb{R}^{n N}\right), x(0) \in$ $D(\mathscr{J})$, and $\mathscr{B} x(0)=u(0)$ the following balance equation is satisfied:

$$
\frac{1}{2} \frac{d}{d t}\|x(t)\|^{2}=\left(\begin{array}{ll}
u^{T}(t) & y^{T}(t)
\end{array}\right) P_{W}\left(\begin{array}{c}
u(t) \\
y(t)
\end{array}\right),
$$


where $P_{W}$ is given by

$$
\begin{aligned}
& \frac{1}{4}\left(\begin{array}{cc}
S^{-T}\left(\tilde{P}_{1}^{2}-\tilde{P}_{1} V V^{T} \tilde{P}_{1}\right) S^{-1} & -2 S^{-T} \tilde{P}_{1} V \tilde{P}_{2} S_{2}^{-1} \\
-2 S_{2}^{-T} \tilde{P}_{2} V^{T} \tilde{P}_{1} S^{-1} & S_{2}^{-T}\left(-\tilde{P}_{2}^{2}+\tilde{P}_{2} V^{T} V \tilde{P}_{2}\right) S_{2}^{-1}
\end{array}\right), \\
& \text { and } \tilde{P}_{1}=\left(I+V V^{T}\right)^{-1}, \quad \tilde{P}_{2}=\left(I+V^{T} V\right)^{-1} \text {. }
\end{aligned}
$$

For more information see [1] and [2].

\section{IMPEDANCE ENERGY-PRESERVING SYSTEMS}

Here we use the term 'impedance energy-preserving system' in the sense of [3]. In that paper the author shows that an impedance energy-preserving system satisfies the relation

$$
\frac{1}{2} \frac{d}{d t}\|x(t)\|^{2}=u(t)^{T} y(t)
$$

for $u \in C^{2}\left((0, \infty) ; \mathbb{R}^{n N}\right), x(0) \in D(\mathscr{J})$ and, $\mathscr{B} x(0)=u(0)$. In [1] the authors show that for an impedance energypreserving system we have $V^{T} V=V V^{T}=I, A_{W}^{*}=-A_{W}$ and $D\left(A_{W}\right)=D\left(A_{W}^{*}\right)$. In [2] it is shown that, in this case, the inputs can be described by

$$
u=\frac{1}{4} S_{2}^{-T}\left[-I-V^{T}, I-V^{T}\right]\left[\begin{array}{l}
f_{\partial} \\
e_{\partial}
\end{array}\right]=W_{\text {imp }}\left[\begin{array}{l}
f_{\partial} \\
e_{\partial}
\end{array}\right]
$$

and the outputs by

$$
y=S_{2}\left[I-V^{T}, \quad-I-V^{T}\right]\left[\begin{array}{l}
f_{\partial} \\
e_{\partial}
\end{array}\right]=C_{\text {imp }}\left[\begin{array}{l}
f_{\partial} \\
e_{\partial}
\end{array}\right] .
$$

Here, the state space is $X=L_{2}\left((a, b) ; \mathbb{R}^{n}\right)$ and the input and output spaces are $U=Y=\mathbb{R}^{n}$. Furthermore, we know that $A_{W}=\mathscr{J}_{\mid D\left(A_{W}\right)}$ is the generator of a contraction semigroup (see Theorem 2.2) with $D\left(A_{W}\right)=\operatorname{ker} \mathscr{B}$.

\section{STATIC FEEDBACK OF AN IMPEDANCE ENERGY-PRESERVING SYSTEM}

In this section we apply feedback (see Figure 1), i.e.,

$$
u=r-\alpha y,
$$

where $r, u, y \in \mathbb{R}^{n N}$ and $\alpha>0$ is a positive definite matrix.

We have that the plant is described by equations (1a)-(1c), where $\mathscr{B} x(t)$ is given by (13), $\mathscr{C} x(t)$ is given by (14) and the differential operator $\mathscr{J}$ when restricted to $D(\mathscr{J}) \cap \operatorname{ker}(\mathscr{B})$ generates a $\mathrm{C}_{0}$-semigroup.

Using the feedback control (15) we can see that the closedloop system is now described by

$$
\begin{gathered}
\dot{x}(t)=\mathscr{J} x(t) \\
\left(W_{\mathrm{imp}}+\alpha C_{\mathrm{imp}}\right)\left[\begin{array}{l}
f_{\partial}(t) \\
e_{\partial}(t)
\end{array}\right]=(\mathscr{B}+\alpha \mathscr{C}) x(t)=r(t) \\
\mathscr{C} x(t)=y(t) .
\end{gathered}
$$

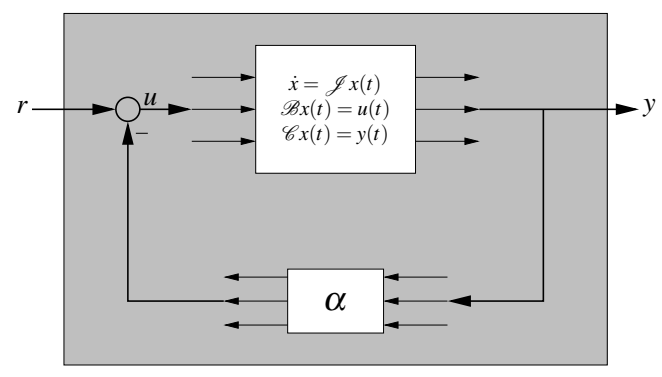

Fig. 1. General control system.
Lemma 4.1: The system described by (16) (with $V V^{T}=I$ ) is a boundary control system. Furthermore, the operator $A_{s}=\mathscr{J}_{\mid D\left(A_{s}\right)}$ generates a contraction semigroup on $X=$ $L_{2}\left((a, b) ; \mathbb{R}^{n}\right)$, where

$$
\begin{gathered}
D\left(A_{s}\right)=\left\{x \in D(\mathscr{J}) \mid\left[\begin{array}{l}
f_{\partial} \\
e_{\partial}
\end{array}\right] \in \operatorname{ker} \widetilde{W}\right\} \quad \text { and } \\
\widetilde{W}=\left(W_{\text {imp }}+\alpha C_{\text {imp }}\right)
\end{gathered}
$$

is a full rank $n N \times 2 n N$ matrix.

Proof: First observe that (18) follows from (16). From Theorem 2.2 we can see that if $\widetilde{W}$ satisfies $\widetilde{W} \Sigma \widetilde{W}^{T} \geq 0(\Sigma$ given by (5)), then we will be done. Since $V$ is unitary we can use Lemma A-1 to show that $\widetilde{W}$ satisfies $\widetilde{W} \Sigma \widetilde{W}^{T}=\alpha+\alpha^{T}>$ 0 (since $\alpha>0$ ).

Remark 4.2: Using Lemma A.1 of [1] we can see that (18) can be factorized as

$$
\tilde{W}=\tilde{S}[I+\tilde{V}, \quad I-\tilde{V}] .
$$

Furthermore, from step 1 and 2 of the proof of Theorem 4.3 of [1] we can show that

$\left\langle A_{s} x, x\right\rangle<0, \forall x \in D\left(A_{s}\right), \quad\left\langle A_{s}^{*} x, x\right\rangle<0, \forall x \in D\left(A_{s}^{*}\right)$. (19b)

Remark 4.3: Following the same procedure used to prove (10)-(11), see [1, p.19], we can show that if $r(t)=0$, $x(0) \in D(\mathscr{J})$, and $(\mathscr{B}+\alpha \mathscr{C}) x(0)=0$, then

$$
\frac{1}{2} \frac{d}{d t}\|x(t)\|^{2}=-y(t)^{T} \alpha y(t) .
$$

Next we study stability of the closed-loop system.

Theorem 4.4: Assume that $\left(\lambda-A_{s}\right)^{-1}: X \rightarrow X$ is a compact operator for $\lambda>0$. Then the system described by (16) (with $V V^{T}=I$ and $r=0$ ), is asymptotically stable.

Remark 4.5: It can be said that most of the examples encountered in the literature satisfy the assumption in the theorem above, see, e.g., [5, p. 269].

Proof: [Proof of Theorem 4.4] By Lemma 4.1 we know that $A_{s}$ generates a contraction semigroup. Thus, for any $x(0) \in X$, the solution $x(t)=T(t) x(0)$ (classical or weak) is bounded in $X$. Since $\left(\lambda-A_{s}\right)^{-1}$ is a compact operator for $\lambda>0$, it follows that the trajectory of the solution $x(t)$, i.e. the set $\gamma(x(0))=\{x(t) \in X, t \geq 0\}$ is precompact in $X$, see Theorem 3.65 of [5]. It then follows that the $w$-limit set ${ }^{1}$ $w(x(0))$ of the trajectory is nonempty, compact, and we have $x(t) \rightarrow w(x(0))$ as $t \rightarrow \infty$, see Theorem 3.61 of [5].

Next we show that $w(x(0))$ contains only the point zero. First we prove this for $x(0) \in D\left(A_{s}\right)$. In this case we have that $x(t)=T(t) x(0) \in D\left(A_{s}\right)$ for all $t \geq 0$, see Theorem 2.1.10 of [4]. Define the energy function

$$
E(t)=\frac{1}{2}\|x(t)\|^{2} .
$$

Since $x(0) \in D\left(A_{s}\right)$ we have that $x(t)$ is differentiable, see [4, $\S 2.1]$. Thus the derivative of $E(t)$ is given by (20) with $r(t)=$ 0 , that is $\dot{E}(t)=\frac{1}{2} \frac{d}{d t}\|x(t)\|^{2}=-y(t)^{T} \alpha y(t)$. Since $\alpha$ is a positive definite matrix we have that $\dot{E}(t)<0$, which shows that $E(t)$ is a Lyapunov function, see Definition 3.62 of [5]. Observe that $\dot{E}(t)=0$ implies $y(t)=0$. Now consider the

\footnotetext{
${ }^{1}$ The $w$-limit set of $x$ is given by $w(x)=\{y \in F \mid y=$ $\lim _{n \rightarrow \infty} T\left(t_{n}\right) x$ with $t_{n} \rightarrow \infty$ as $\left.n \rightarrow \infty\right\}$, where $F$ is a closed subset of $X$.
} 
set $\{x \in X \mid \dot{E}(t)=0\}$ or equivalently $\mathscr{O}=\{x \in X \mid y(t)=0\}$ and let $\mathscr{E}$ be its largest invariant subset. Since $\gamma(x(0))$ is precompact, it follows from LaSalle's principle that $x(t) \rightarrow \mathscr{E}$ as $t \rightarrow \infty$, see Theorem 3.64 of [5]. We show that $\mathscr{E}=\{0\}$. Since $x(0)$ and $x(t)$ belong to $D\left(A_{s}\right)$, we have that $\mathscr{E} \subset$ $D\left(A_{S}\right)$, see [5, p.270]. Let $\tilde{x} \in \mathscr{E}$ and let $z(t)=T(t) \tilde{x}$ be the corresponding solution. Since $\mathscr{E}$ is invariant we have that $z(t) \in \mathscr{E}$ for $t \geq 0$. Recall that all $x \in \mathscr{O}$ satisfy $y(t)=0$. It then follows that $z(t)$ is the solution of $\dot{z}(t)=\mathscr{J} z(t)$ satisfying $r(t)=y(t)=0$. Since this is a PDE with all boundary variables set to zero, we must have that the only solution is $z(t)=0$ for $t \geq 0$. Hence $\tilde{x}=0$; and thus, $\mathscr{E}=\{0\}$. Altogether means that $x(t) \rightarrow 0$ as $t \rightarrow \infty$ for any $x(0) \in D\left(A_{s}\right)$.

The same statement holds for $x(0) \in X$, see [5, p.270].

\section{A. First order differential operator (case $N=1$ )}

In this section we study the differential operator

$$
\mathscr{J} x=P_{0} x(z)+P_{1} \frac{d x}{d z}(z) .
$$

This case includes the well-known beam and wave equations. We assume that the input and outputs have been chosen so that the resulting system is impedance energy-preserving, i.e., $\frac{1}{2} \frac{d}{d t}\|x(t)\|^{2}=u(t)^{T} y(t)$ holds. We want to apply static feedback to the resulting BCS, see Figure 1. We already know that, in this case, the closed-loop system is a BCS and that the operator $A_{s}=\mathscr{J}_{\mid D\left(A_{s}\right)}$ generates a contraction semigroup, see Lemma 4.1. We want to check whether the closed-loop system is asymptotically stable. To do so we need to check that the resolvent $\left(\lambda-A_{s}\right)^{-1}$ is compact for $\lambda>0$, see Theorem 4.4 .

First we study the eigenvalues of $A_{s}$. From (19b) we know that $\left\langle A_{s} x, x\right\rangle<0$ for all $x \in D\left(A_{s}\right)$. Let $x_{1}$ be any eigenvector of $A_{s}$ with corresponding eigenvalue $\lambda$. Then we have that

$$
\operatorname{Re}\left\langle A_{s} x_{1}, x_{1}\right\rangle=\operatorname{Re}\left\langle\lambda x_{1}, x_{1}\right\rangle=\operatorname{Re} \lambda\left\|x_{1}\right\|^{2}<0 .
$$

This implies that all eigenvalues of $A_{s}$ satisfy $\operatorname{Re} \lambda<0$. Moreover, we have that this eigenvector $x_{1}$ is the solution of

$$
P_{0} x_{1}+P_{1} \frac{d x_{1}}{d z}=\lambda x_{1} \Longleftrightarrow \frac{d x_{1}}{d z}=P_{1}^{-1}\left(\lambda-P_{0}\right) x_{1} .
$$

The general solution of the equation above is given by

$$
x_{1}(z)=\mathrm{e}^{P_{1}^{-1}\left(\lambda-P_{0}\right)(z-a)} c
$$

where $c$ is a constant vector. Using (19) and the boundary conditions on $D\left(A_{s}\right)$, see (17), we get

$$
\begin{gathered}
\tilde{W}\left[\begin{array}{l}
f_{\partial} \\
e_{\partial}
\end{array}\right]=\tilde{W} R_{\text {ext }}\left[\begin{array}{l}
x_{1}(b) \\
x_{1}(a)
\end{array}\right]=0 \\
\Longleftrightarrow \tilde{S}[I+\tilde{V}, \quad I-\tilde{V}]\left[\begin{array}{cc}
P_{1} & -P_{1} \\
I & I
\end{array}\right]\left[\begin{array}{l}
x_{1}(b) \\
x_{1}(a)
\end{array}\right]=0
\end{gathered}
$$

where we used Definition 2.1 and (19a). Using (23) in the above equation gives

$$
\begin{aligned}
\left(\left[(I+\tilde{V}) P_{1}+(I-\tilde{V})\right]\right. & \mathrm{e}^{P_{1}^{-1}\left(\lambda-P_{0}\right)(b-a)} \\
& \left.+\left[-(I+\tilde{V}) P_{1}+(I-\tilde{V})\right]\right) c=0 .
\end{aligned}
$$

We know that $\lambda$ is an eigenvalue of $A_{s}$ iff the matrix above is singular. At the beginning of this subsection we showed that the eigenvalues of $A_{s}$ satisfy $\operatorname{Re} \lambda<0$, which shows that the matrix above is nonsingular if $\operatorname{Re} \lambda \geq 0$, otherwise $A_{s}$ would have eigenvalues with $\operatorname{Re} \lambda \geq 0$. In summary we have the following result.

Lemma 4.6: Consider the system described in Lemma 4.1 with $\mathscr{J}$ given by (22). Then the eigenvalues of $A_{s}$ satisfy $\operatorname{Re} \lambda<0$. Furthermore, if $\lambda \geq 0$ the matrix on (25) is nonsingular and $\left(\lambda-A_{s}\right)^{-1}$ is a compact operator.

Proof: That the eigenvalues of $A_{s}$ satisfy $\operatorname{Re} \lambda<0$ and that the matrix is nonsingular was proved above.

Next we study the resolvent of $A_{s}$. First we study the range of $\left(\lambda-A_{s}\right): D\left(A_{s}\right) \rightarrow X$ for $\lambda \geq 0$. To see this, consider $\left(\lambda-A_{s}\right) x=y$, which is equivalent to solve

$$
\frac{d x}{d z}(z)=P_{1}^{-1}\left(\lambda-P_{0}\right) x(z)-P_{1}^{-1} y(z)
$$

for $x \in D\left(A_{s}\right)$. The general solution of (26) is given by

$$
x(z)=\mathrm{e}^{P_{1}^{-1}\left(\lambda-P_{0}\right)(z-a)} c-\int_{a}^{z} \mathrm{e}^{P_{1}^{-1}\left(\lambda-P_{0}\right)(z-\tau)} P_{1}^{-1} y(\tau) d \tau
$$

with $c$ a constant vector. Since $x \in D\left(A_{s}\right)$ the boundary conditions (see (17)) are given by (24). Using (27) in (24) yields

$$
\begin{aligned}
& \left(\left[(I+\tilde{V}) P_{1}+(I-\tilde{V})\right] \mathrm{e}^{P_{1}^{-1}\left(\lambda-P_{0}\right)(b-a)}\right. \\
& \left.+\left[-(I+\tilde{V}) P_{1}+(I-\tilde{V})\right]\right) c= \\
& \quad\left[(I+\tilde{V}) P_{1}+(I-\tilde{V})\right] \int_{a}^{b} \mathrm{e}^{P_{1}^{-1}\left(\lambda-P_{0}\right)(b-\tau)} P_{1}^{-1} y(\tau) d \tau .
\end{aligned}
$$

We already showed that when $\operatorname{Re} \lambda \geq 0$ the matrix on the LHS is nonsingular. In that case, $c$ can be defined uniquely, which implies that (26) has a unique solution in $D\left(A_{s}\right)$. This solution is given by (27). Hence $\left(\lambda-A_{s}\right)^{-1}$ exists when $\operatorname{Re} \lambda \geq 0$. In order to prove that (27) defines the resolvent of $A_{s}$ we need to show that it is bounded. First observe that the dimension of the domain of $\mathrm{e}^{P_{1}^{-1}\left(\lambda-P_{0}\right)(z-a)} c$ is finite, thus it is a compact operator (hence bounded), see [6, Th. 2.6-9 b, Th. 8.1-4, Th. 8.1-2 b]. Also, the integral operator on (27) is compact, see Theorem A.3.52 of [4]. Then, $\left(\lambda-A_{s}\right)^{-1}$ given by (27) is the sum of two compact operators (see see [6, p. 407]), hence $\left(\lambda-A_{s}\right)^{-1}$ is a compact operator when $\operatorname{Re} \lambda \geq 0$.

From the Lemma above and Theorem 4.4 we know that this type of closed-loop systems (when $N=1$ ) are asymptotically stable. Also, if $P_{0}=0$ we can see that when $\lambda$ is a pure imaginary number, the resolvent (27) is clearly a uniformly bounded operator since $P_{1}$ has only real eigenvalues and hence the magnitude of $\mathrm{e}^{\lambda P_{1}^{-1} z}$ is equal to one. Since $A_{s}$ generates a contraction semigroup it follows that the conditions on Corollary 3.36 of [5] are satisfied, which proves that, in this case, the closed-loop is exponentially stable.

\section{DYNAMIC FEEDBACK OF AN IMPEDANCE ENERGY-PRESERVING SYSTEM}

In this section we generalize the class of static controllers described in the previous section. More precisely, we replace 
the static matrix $\alpha$ with a matrix transfer function $\alpha(s)$ where $s \in \mathbb{C}$ is a complex variable.

The state space representation of the controller is given by

$$
\begin{aligned}
\dot{v}(t) & =A_{\alpha} v(t)+B_{\alpha} y(t) \\
y_{\alpha}(t) & =C_{\alpha} v(t)+D_{\alpha} y(t)
\end{aligned}
$$

where $v \in \mathbb{R}^{m}$ is the state of the minimal realization. In this way, equation (15) becomes

$$
u=r-y_{\alpha}=r-C_{\alpha} v-D_{\alpha} \mathscr{C} x,
$$

where (1c) was used.

In the literature, an $n \times n$ rational matrix $H(s)$ is said to be positive real (PR) if: i) all elements of $H(s)$ are analytic in the open right-half plane $\operatorname{Re}(s)>0$, ii) poles of any element of $H(s)$ on the $j w$-axis are distinct, and the associated residue matrix of $H(s)$ is $\geq 0$, iii) $H(j w)+H^{T}(-j w) \geq 0 \forall w$ which is not a pole of any element of $H(j w)$.

Definition 5.1 (Tao and Ioannou [7]): A rational matrix $H(s)$ is strictly positive real (SPR) if $H(s-\varepsilon)$ is positive real (PR) for some $\varepsilon>0$.

The next lemma is used in the stability analysis (see [7]).

Lemma 5.2: Assume that the transfer matrix $H(s)$ has all its poles in $\operatorname{Re}(s)<-\gamma$, where $\gamma>0$ and $(A, B, C, D)$ is a minimal realization of $H(s)$. Then $H(s-\gamma)$ is PR if and only if there exist matrices $P, Q$ and $K$ such that $P=P^{T}>0$ and

$P A+A^{T} P=-Q Q^{T}-2 \gamma P ; P B=C^{T}-Q K ; K^{T} K=D+D^{T}$.

Throughout this section the controller is assumed to be SPR. Let $x \in X$ be the state of the plant, $v \in \mathbb{R}^{m}$ the state of the controller, and $w=\left[\begin{array}{l}x \\ v\end{array}\right]$. Using the feedback control (29) and the fact that $u(t)=\mathscr{B} x(t)$, see (1b), we can see that the closed-loop system is now described by

$$
\begin{gathered}
\dot{w}(t)=\mathscr{J}_{c} w(t), \quad w(0) \in \tilde{X} \\
{\left[\mathscr{B}+D_{\alpha} \mathscr{C}, \quad C_{\alpha}\right] w(t)=r(t)} \\
{\left[\begin{array}{cc}
\mathscr{C} & 0
\end{array}\right] w(t)=y(t),}
\end{gathered}
$$

where $\tilde{X}=\left[\begin{array}{c}X \\ \mathbb{R}^{m}\end{array}\right]$ is the state space of the closed-loop system, $w=\left[\begin{array}{l}x \\ v\end{array}\right] \in \tilde{X}$, and $\mathscr{J}_{c}: \tilde{X} \rightarrow \tilde{X}$ is a linear operator defined as

$$
\mathscr{J}_{c} w=\left[\begin{array}{cc}
\mathscr{J} & 0 \\
B_{\alpha} \mathscr{C} & A_{\alpha}
\end{array}\right]\left[\begin{array}{l}
x \\
v
\end{array}\right]
$$

with $D\left(\mathscr{J}_{c}\right)=D(\mathscr{J}) \oplus \mathbb{R}^{m}$. The inner product on the space $\tilde{X}$ is defined as

$$
\left\langle w_{1}, w_{2}\right\rangle_{\tilde{X}}=\left\langle x_{1}, x_{2}\right\rangle_{X}+\frac{1}{2} v_{1}^{T} P v_{2}+\frac{1}{2} v_{2}^{T} P v_{1},
$$

where $P$ is the positive definite matrix found in Lemma 5.2.

Lemma 5.3: Let the state of the open-loop system of Figure 1 satisfy $\frac{1}{2} \frac{d}{d t}\|x(t)\|^{2}=u(t)^{T} y(t)$ and the controller $\alpha(s)$ be SPR. Then the system (31)-(32) is a boundary control system. Furthermore, the operator $A_{c}$ defined by

$$
A_{c} w=\left[\begin{array}{cc}
\mathscr{J} & 0 \\
B_{\alpha} \mathscr{C} & A_{\alpha}
\end{array}\right]\left[\begin{array}{l}
x \\
v
\end{array}\right]
$$

with

$$
D\left(A_{c}\right)=\left\{\left[\begin{array}{l}
x \\
v
\end{array}\right] \in\left[\begin{array}{c}
X \\
\mathbb{R}^{m}
\end{array}\right] \mid x \in D(\mathscr{J}) \text {, and }\left[\begin{array}{c}
f_{\partial} \\
e_{\partial} \\
v
\end{array}\right] \in \operatorname{ker} \widetilde{W}_{D}\right\},
$$

where

$$
\widetilde{W}_{D}=\left[\left(W_{\mathrm{imp}}+D_{\alpha} C_{\mathrm{imp}}\right), C_{\alpha}\right],
$$

generates a contraction semigroup.

Proof: First we need to prove that there exists an operator $B \in \mathscr{L}(U, \tilde{X})$ such that for all $r \in U, B r \in D(\mathscr{J}) \times$ $\mathbb{R}^{m}$, and $\left[\mathscr{B}+D_{\alpha} \mathscr{C}, C_{\alpha}\right] B r=r$. From the proof of Theorem 4.5 of [1] we know that if the matrix $\tilde{W}_{D}$ has full rank, then such operator $B$ exists. Thus we need to prove that $\tilde{W}_{D}$ has full rank. Since the open-loop system is assumed to be impedance energy-preserving, we must have $V V^{T}=I$. From this and Lemma A-2 we can see that the matrix $\left(W_{\mathrm{imp}}+D_{\alpha} C_{\mathrm{imp}}\right)$ has full row-rank. Hence, we can conclude that $\tilde{W}_{D}$ in (34c) has also full row-rank.

Equations (34) follow easily from (31) and the proof of Lemma 4.1. Next we need to prove that $A_{c}$ generates a semigroup. We will use the Lumer-Phillips theorem (see Theorem 2.27 of [5]). First we prove that $\left\langle A_{c} w, w\right\rangle \leq 0$. Let $w=\left[\begin{array}{l}x \\ v\end{array}\right] \in D\left(A_{c}\right)$, then we have

$$
\begin{aligned}
&\left\langle A_{c} w, w\right\rangle_{\tilde{X}}=\langle\mathscr{J} x, x\rangle_{X}+\frac{1}{2}\left(A_{\alpha} v+B_{\alpha} y\right)^{T} P v \\
&+\frac{1}{2} v^{T} P\left(A_{\alpha} v+B_{\alpha} y\right) \\
&=\langle\mathscr{J} x, x\rangle_{X}+\frac{1}{2} v^{T}\left(A_{\alpha}^{T} P+P A_{\alpha}\right) v+\frac{1}{2} y^{T} B_{\alpha}^{T} P v+\frac{1}{2} v^{T} P B_{\alpha} y .
\end{aligned}
$$

From Equation (4.8) of [1] and Lemma 5.2 we obtain

$$
\begin{aligned}
= & \frac{1}{2}\left[\begin{array}{rr}
f_{\partial}^{T} & e_{\partial}^{T}
\end{array}\right] \Sigma\left[\begin{array}{c}
f_{\partial} \\
e_{\partial}
\end{array}\right]+\frac{1}{2} v^{T}\left(-Q Q^{T}-2 \gamma P\right) v \\
& +\frac{1}{2} y^{T}\left(C_{\alpha}-K^{T} Q^{T}\right) v+\frac{1}{2} v^{T}\left(C_{\alpha}^{T}-Q K\right) y .
\end{aligned}
$$

Using (A-1) together with (13) and (14) yields

$$
\begin{aligned}
=\frac{1}{2} y^{T} u+\frac{1}{2} u^{T} y+\frac{1}{2} v^{T}\left(-Q Q^{T}-2 \gamma P\right) v \\
\quad+\frac{1}{2} y^{T}\left(C_{\alpha}-K^{T} Q^{T}\right) v+\frac{1}{2} v^{T}\left(C_{\alpha}^{T}-Q K\right) y .
\end{aligned}
$$

Since $w=\left[\begin{array}{l}x \\ v\end{array}\right] \in D\left(A_{c}\right)$ we have that $C_{\alpha} v=-\left(W_{\text {imp }}+\right.$ $\left.D_{\alpha} C_{\text {imp }}\right)\left[\begin{array}{l}f_{\partial} \\ e_{\partial}\end{array}\right]$, see (34), and using again (13) and (14) gives, after simplification

$$
\begin{gathered}
=\frac{1}{2} v^{T}\left(-Q Q^{T}-2 \gamma P\right) v-\frac{1}{2} y^{T} D_{\alpha} y-\frac{1}{2} y^{T} D_{\alpha}^{T} y \\
-\frac{1}{2} y^{T} K^{T} Q^{T} v-\frac{1}{2} v^{T} Q K y
\end{gathered}
$$

and using again Lemma 5.2 yields

$$
\left\langle A_{c} w, w\right\rangle_{\tilde{X}}=-\gamma v^{T} P v-\frac{1}{2}\left(K y+Q^{T} v\right)^{T}\left(K y+Q^{T} v\right) .
$$

Since $\gamma>0$ and $P$ is positive definite it thus follows from the equation above that $\left\langle A_{c} w, w\right\rangle_{\tilde{X}} \leq 0$. 
Next we need to prove that the range of $\left(I-A_{c}\right)$ is equal to $\tilde{X}$. In order to do so, we can show that for all $\left[\begin{array}{c}f \\ z\end{array}\right] \in\left[\begin{array}{c}X \\ \mathbb{R}^{m}\end{array}\right]$ there exists $\left[\begin{array}{l}x \\ v\end{array}\right] \in D\left(A_{c}\right)$ such that

$$
\left[\begin{array}{c}
f \\
z
\end{array}\right]=\left[\begin{array}{c}
(I-\mathscr{J}) x \\
-B_{\alpha} \mathscr{C} x+\left(I-A_{\alpha}\right) v
\end{array}\right] .
$$

Observe that since $\left[\begin{array}{l}x \\ v\end{array}\right] \in D\left(A_{c}\right)$ we must have (see (31))

$$
\left(\mathscr{B}+D_{\alpha} \mathscr{C}\right) x+C_{\alpha} v=0 .
$$

We need to solve (36) and (37) for $\left[\begin{array}{l}f \\ z\end{array}\right] \in\left[\begin{array}{c}X \\ \mathbb{R}^{m}\end{array}\right]$ given. Recall that $A_{\alpha}$ is assumed to have only negative eigenvalues, and hence $\left(I-A_{\alpha}\right)$ is a nonsingular matrix. Using the lower equation of (36) into (37) yields

$$
(\mathscr{B}+\alpha(1) \mathscr{C}) x=-C_{\alpha}\left(I-A_{\alpha}\right)^{-1} z,
$$

where $\alpha(1)=C_{\alpha}\left(I-A_{\alpha}\right)^{-1} B_{\alpha}+D_{\alpha}$. We now need to find an $x$ that satisfies (38) and the upper equation in (36). In these two equations let $\tilde{z}=-C_{\alpha}\left(I-A_{\alpha}\right)^{-1} z$ and $x=x_{\text {new }}+\tilde{B} \tilde{z}$ where $\tilde{B}$ is such that $(\mathscr{B}+\alpha(1) \mathscr{C}) \tilde{B}=I$ (the existence of $\tilde{B}$ is proved in [1]). This gives

$$
\begin{aligned}
(I-\mathscr{J}) x_{\text {new }} & =f-(I-\mathscr{J}) \tilde{B} \tilde{z} \\
(\mathscr{B}+\alpha(1) \mathscr{C}) x_{\text {new }} & =0 .
\end{aligned}
$$

Following the proof of Theorem 4.1 it is not difficult to see that if (40) holds then $\mathscr{J}$ generates a contraction semigroup. This implies that $(I-\mathscr{J})$ has an inverse and hence $x_{\text {new }}$ exists. Thus, for $\left[\begin{array}{l}f \\ z\end{array}\right] \in\left[\begin{array}{c}X \\ \mathbb{R}^{m}\end{array}\right]$ we can find $\left[\begin{array}{l}x \\ v\end{array}\right] \in D\left(A_{c}\right)$ such that (36) and (37) holds.

In the rest of this section we denote by $A_{\lambda}$ the operator $A_{s}$ described in Lemma 4.1 with $\alpha$ replaced by $\alpha(\lambda)=C_{\alpha}(\lambda I-$ $\left.A_{\alpha}\right)^{-1} B_{\alpha}+D_{\alpha}$ in (18). Next we show that if the resolvent of $A_{\lambda}$ is compact for $\lambda>0$, then the associated closed-loop system will also have a compact resolvent.

Theorem 5.4: Consider the system described in Theorem 2.2. Let the energy of this system satisfy $\frac{1}{2} \frac{d}{d t}\|x(t)\|^{2}=$ $u(t)^{T} y(t)$. Assume that $\alpha(s)$ is a SPR function and that the resolvent of the operator $A_{\lambda}$ described above is compact for $\lambda>0$. Then $\left(\lambda I-A_{c}\right)^{-1}$ is also compact for $\lambda>0$.

Proof: We will use Theorem $8.1-3$ of [6], which states that an operator is compact iff it maps every bounded sequence onto a sequence which has a convergent subsequence. First we find the inverse of $\left(\lambda I-A_{c}\right)$ for $\lambda>0$ by following the same procedure used to find (36)-(40). We know that this inverse exists since $A_{c}$ generates a contraction semigroup. From (39)-(40) we see, in this case, that $x_{\text {new }} \in D\left(A_{\lambda}\right)$ and $x_{\text {new }}=\left(I-A_{\lambda}\right)^{-1} f-\left(I-A_{\lambda}\right)^{-1}(I-\mathscr{J}) \tilde{B} \tilde{z}$, where $\tilde{z}=$ $-C_{\alpha}\left(I-A_{\alpha}\right)^{-1} z$. Since $x=x_{\text {new }}+\tilde{B} \tilde{z}$ we obtain that

$$
x=\left(I-A_{\lambda}\right)^{-1} f-\left(I-A_{\lambda}\right)^{-1}(I-\mathscr{J}) \tilde{B} \tilde{z}+\tilde{B} \tilde{z},
$$

and from the lower equation of (36) we get

$$
v=\left(I-A_{\alpha}\right)^{-1} B_{\alpha} \mathscr{C} x+\left(I-A_{\alpha}\right)^{-1} z .
$$

Let $\left\{k_{n}\right\}=\left\{\left[\begin{array}{c}f_{n} \\ z_{n}\end{array}\right]\right\} \in \tilde{X}=\left[\begin{array}{c}X \\ \mathbb{R}^{m}\end{array}\right]$ be any bounded sequence in $\tilde{X}$ and let $w_{n}=\left[\begin{array}{l}x_{n} \\ v_{n}\end{array}\right] \in D\left(A_{c}\right)$ such that $w_{n}=\left(\lambda I-A_{c}\right)^{-1} k_{n}$. By Theorem 5.3 we know that $A_{c}$ generates a contraction semigroup and by the Hille-Yosida theorem it follows that $\left\|\left(\lambda I-A_{c}\right)^{-1}\right\| \leq \frac{1}{\lambda}$ for $\lambda>0$. Hence the sequence $\left\{w_{n}\right\}$ is bounded too. Since we know that $\left(\lambda I-A_{\lambda}\right)^{-1}$ is compact and that $\mathscr{J} \tilde{B}$ is bounded (see Definition 3.3.2 of [4]), we have that $\left\{x_{n}\right\}$ has a convergent subsequence, see (41). Also, since $\left\{v_{n}\right\}$ is bounded and belongs to a finite dimensional subspace of $\tilde{X}$, it follows that $\left\{v_{n}\right\}$ has another convergent subsequence. Hence, $w_{n}=\left[\begin{array}{l}x_{n} \\ v_{n}\end{array}\right]$ has a convergent subsequence and therefore $\left(\lambda I-A_{c}\right)^{-1}$ is compact for $\lambda>0$.

Next we give an asymptotic stability result similar to the one in Theorem 4.4.

Theorem 5.5: Consider the system given by (31). Let the transfer function $\alpha(s)$ be a SPR function. Let the resolvent associated with $A_{\lambda}$ be compact for $\lambda>0$. Then the system described by (31) with $r=0$, is asymptotically stable.

Proof: The proof is similar to that of Theorem 4.4. First we prove this for $w(0) \in D\left(A_{c}\right)$. By Lemma 4.1 we know that $A_{c}$ generates a contraction semigroup. In this case we have that $w(t)=T_{c}(t) x(0) \in D\left(A_{c}\right)$ for all $t \geq 0$, see Theorem 2.1.10 of [4]. Define the energy function

$$
E_{c}(t)=\frac{1}{2}\|w(t)\|_{\tilde{X}}^{2}=\frac{1}{2}\langle w(t), w(t)\rangle_{\tilde{X}}
$$

Since $w(0) \in D\left(A_{c}\right)$ we have that $w(t)$ is differentiable, see $[4, \S 2.1]$. By differentiating the equation above and using (31) and (35) we obtain

$$
\begin{aligned}
& \dot{E}_{c}(t)=\langle\dot{w}(t), w(t)\rangle_{\tilde{X}}=\left\langle A_{c} w(t), w(t)\right\rangle_{\tilde{X}} \\
& =-\gamma v(t)^{T} P v(t)-\frac{1}{2}\left(K y(t)+Q^{T} v(t)\right)^{T}\left(K y(t)+Q^{T} v(t)\right),
\end{aligned}
$$

where $\gamma>0$ and $P$ is positive definite. Since $\left(\lambda I-A_{\lambda}\right)^{-1}$ is compact, it follows from Theorem 5.4 that $\left(\lambda I-A_{c}\right)^{-1}$ is also compact. Since $\left(\lambda I-A_{c}\right)^{-1}$ is compact and $T_{c}(t)$ is a contraction, it follows from LaSalle's principle that all solutions of (31) asymptotically tend to the maximal invariant set of $\mathscr{O}_{c}=\left\{x \in X \mid \dot{E}_{c}(t)=0\right\}$. Let $\mathscr{E}$ be the largest invariant subset of $\mathscr{O}_{c}$. Next we show that $\mathscr{E}=\{0\}$. The condition $\dot{E}_{c}(t)=0$ implies, from (44), that $v(t)=0$; and hence, $\dot{v}(t)=$ 0 . Then by (28) we must have that $B_{\alpha} y(t)=0$. Since $\alpha(s)$ is SPR, we have that $\alpha(j w)+\alpha^{T}(-j w)>0$. This implies that

$$
\begin{aligned}
& y^{T}(t)\left[\alpha(j w)+\alpha^{T}(-j w)\right] y(t)>0 \\
\Rightarrow & y^{T}(t)\left[D_{\alpha}+D_{\alpha}^{T}\right] y(t)>0 \Rightarrow K^{T} K>0 .
\end{aligned}
$$

In the second step the facts $\alpha(j w)=C_{\alpha}\left(j w-A_{\alpha}\right)^{-1} B_{\alpha}+D_{\alpha}$ and $B_{\alpha} y(t)=0$ were used, and in the third step we used (30). Since $v(t)=0$ and $K^{T} K>0$ it follows from (44) that $y(t)=0$, and hence by (28) we also obtain $y_{\alpha}(t)=0$.

Therefore from (31) and (34) it follows that the invariant solution of (31) in $\mathscr{O}_{c}$ reduces to the invariant solution of the associated open-loop system in the domain (17)-(18). The rest of the proof follows from the proof of Theorem 4.4.

\section{SCATTERING ENERGY-PRESERVING SYSTEMS}

Here we use the term 'scattering energy-preserving system' in the sense of [3]. In that paper the author shows that a scattering energy-preserving system satisfies the relation

$$
\frac{1}{2} \frac{d}{d t}\|x(t)\|^{2}=\|u(t)\|^{2}-\|y(t)\|^{2}
$$


for $u \in C^{2}\left((0, \infty) ; \mathbb{R}^{n N}\right), x(0) \in D(\mathscr{J})$ and, $\mathscr{B} x(0)=u(0)$. In [2] it is shown that, in this case, $V=0, \tilde{P}_{1}=\tilde{P}_{2}=I$, $S^{-T} S^{-1}=4 I$, and $S_{2}^{-T} S_{2}^{-1}=4 I$. This means that $W$ has the form $W=S\left[\begin{array}{ll}I & I\end{array}\right]$.

For this type of systems related to the operator $\mathscr{J}$ we have the following result.

Theorem 6.1: Consider the system described in Theorem 2.2 with $\mathscr{J} e=P_{1} \frac{d e}{d z}(z)$ and $\frac{1}{2} \frac{d}{d t}\|x(t)\|^{2}=\|u(t)\|^{2}-$ $\|y(t)\|^{2}$. Then the system is exponentially stable and exactly observable in finite time.

Proof: First observe that $W$ has the form $W=$ $S\left[\begin{array}{ll}I & I\end{array}\right]$. In order to check stability of the system we study the resolvent of $A_{W}=\mathscr{J}_{\mid D\left(A_{W}\right)}$. Let $x \in D\left(A_{W}\right)$ and observe that $y(z)=\left(\lambda-A_{W}\right) x(z)=\lambda x(z)-P_{1} \frac{d x}{d z}(z)$. Thus $\frac{d x}{d z}(z)=\lambda P_{1}^{-1} x(z)-P_{1}^{-1} y(z)$ need to be solved. The general solution is given by

$$
x(z)=\mathrm{e}^{\lambda P_{1}^{-1}(z-a)} c-\int_{a}^{z} \mathrm{e}^{\lambda P_{1}^{-1}(z-\tau)} P_{1}^{-1} y(\tau) d \tau
$$

where $c$ is a constant vector. Since $x \in D\left(A_{W}\right)$ the boundary conditions (see Theorem 2.2) are described by (see (4))

$$
W R_{\text {ext }}\left[\begin{array}{c}
x(b) \\
x(a)
\end{array}\right]=\left[\begin{array}{cc}
P_{1}+I & I-P_{1}
\end{array}\right]\left[\begin{array}{c}
x(b) \\
x(a)
\end{array}\right]=0 .
$$

Using (45) in the equation above yields

$$
\begin{aligned}
\left(P_{1}+I\right) & {\left[\mathrm{e}^{\lambda P_{1}^{-1}(b-a)} c-\int_{a}^{b} \mathrm{e}^{\lambda P_{1}^{-1}(b-\tau)} P_{1}^{-1} y(\tau) d \tau\right] } \\
+ & \left(I-P_{1}\right) c=0 \\
\Longleftrightarrow & {\left[\left(P_{1}+I\right) \mathrm{e}^{\lambda P_{1}^{-1}(b-a)}+\left(I-P_{1}\right)\right] c=} \\
& \left(P_{1}+I\right) \int_{a}^{b} \mathrm{e}^{\lambda P_{1}^{-1}(b-\tau)} P_{1}^{-1} y(\tau) d \tau .
\end{aligned}
$$

It follows from Lemma 14 of [8] that when $\lambda \in \mathbb{C} \backslash \mathbb{R}^{-}$the matrix on the left hand side is nonsingular. In that case, $c$ can be defined uniquely, which implies that $\left(\lambda-A_{W}\right)^{-1}$ exists. Also, it is not difficult to show that $\left(\lambda-A_{W}\right)^{-1}$ is bounded, hence Equation (45) defines the resolvent operator of $A_{W}$.

It is easy to show that the resolvent (45) is uniformly bounded (see the last paragraph of Subsection IV-A). Since $A_{W}$ generates a contraction semigroup it follows from Corollary 3.36 of [5] that the system is exponentially stable. Finally, from Theorem 11.3.8 of [9] one can see that the system is also exactly observable in finite time.

Lemma 6.2: Consider the system of Theorem 2.2 with $\mathscr{J} e=P_{1} \frac{d e}{d z}(z)$ and $\frac{1}{2} \frac{d}{d t}\|x(t)\|^{2}=\|u(t)\|^{2}-\|y(t)\|^{2}$. Then the resolvent $\left(\lambda-A_{W}\right)^{-1}$, with $\lambda \geq 0$, is a compact operator, where $A_{W}=\mathscr{J}_{\mid D\left(A_{W}\right)}$. Furthermore, the operator $\tilde{\mathscr{A}}_{\mathscr{W}} e=$ $P_{0} e(z)+P_{1} \frac{d e}{d z}(z)$ has compact resolvent for $\lambda>0$, i.e., $\left(\lambda-\tilde{A}_{W}\right)^{-1}$ is a compact operator.

Proof: From Theorem 6.1 we know that the resolvent of $A_{W}$ is given by (45) for $\lambda \geq 0$. The integral operator on the RHS is clearly compact, see Theorem A.3.52 of [4]. The exponential operator $\mathrm{e}^{\lambda P_{1}^{-1}(z-a)} c$ is also compact, since it is a finite rank operator, see Theorem 8.1-4 of [6]. Since $\left(\lambda-A_{W}\right)^{-1}$ in (45) is the sum of two compact operators we have that it is also compact for $\lambda \geq 0$, see [6, p. 407].
Using Lemma A-3 we can conclude that $\left(\lambda-\tilde{A}_{W}\right)^{-1}$ is compact for $\lambda>0$ since $\tilde{A}_{W}$ is also the generator of a contraction semigroup.

Theorem 6.3: Consider the system of Theorem 2.2 with $\mathscr{J} e=P_{0} e(z)+P_{1} \frac{d e}{d z}(z)$ and $\frac{1}{2} \frac{d}{d t}\|x(t)\|^{2}=\|u(t)\|^{2}-\|y(t)\|^{2}$. Then the system is globally asymptotically stable.

Proof: From Lemma 6.2 we know that $\left(\lambda-A_{W}\right)^{-1}$, with $\lambda>0$, is a compact operator, where $A_{W}=\mathscr{J}_{\mid D\left(A_{W}\right)}$. The rest of the proof follows similar to the proof of Theorem 4.4, noting that $\dot{E}(t)=-\|y(t)\|^{2}$.

\section{APPENDIX}

Lemma A-1: Let $S_{2}, V \in \mathbb{R}^{n \times n}$ and $R_{\text {ext }}, \Sigma \in \mathbb{R}^{2 n \times 2 n}$ where $\Sigma$ is given by (5). Consider the matrices $W_{\text {imp }}$ and $C_{\text {imp }}$ given by (13) and (14), respectively. If $V$ satisfies $V V^{T}=I=V^{T} V$ (impedance passive system), then we have

$$
\begin{gathered}
C_{\mathrm{imp}}^{T} W_{\mathrm{imp}}+W_{\mathrm{imp}}^{T} C_{\mathrm{imp}}=\Sigma, \\
C_{\mathrm{imp}} C_{\mathrm{imp}}^{T}=4 S_{2} S_{2}^{T}, \quad W_{\mathrm{imp}} W_{\mathrm{imp}}^{T}=\frac{1}{4} S_{2}^{-T} S_{2}^{-1}, \\
C_{\mathrm{imp}} W_{\mathrm{imp}}^{T}=0, \quad \text { and } \quad W_{\mathrm{imp}} C_{\mathrm{imp}}^{T}=0 .
\end{gathered}
$$

Proof: It follows easily by using algebra and $V V^{T}=I$.

Lemma A-2: Consider the matrices $W_{\mathrm{imp}}$ and $C_{\mathrm{imp}}$ given by (13) and (14), respectively. Let $V$ and $D$ be $n \times n$ matrices, with $V V^{T}=I=V^{T} V$. Then, the matrix given by

$$
\widetilde{W}=W_{\text {imp }}+D C_{\text {imp }}
$$

has full row-rank.

Proof: It is easy to show that the matrix $\widetilde{W} \widetilde{W}^{T}$ is nonsingular.

The following lemma is not difficult to prove.

Lemma $A$-3: Let $A=A_{u}+A_{b}$ be a closed and densely defined operator on $X$, where $A_{b}$ is a bounded operator on $X$ and $A_{u}$ is an unbounded operator with $D\left(A_{u}\right)=D(A)$. If $A$ satisfies $\operatorname{Re}\langle A z, z\rangle \leq 0$ and $\left(\lambda-A_{u}\right)^{-1}$ is a compact operator for $\lambda>0$. Then the operator $(\lambda-A)^{-1}$ is a compact operator for $\lambda>0$.

\section{REFERENCES}

[1] Y. Le Gorrec, H. Zwart, and B. Maschke, "Dirac structures and boundary control systems associated with skew-symmetric differential operators," 2004, Internal report No. 1730, University of Twente (available at http://www.math.utwente.nl/publications/).

[2] J.A. Villegas, H. Zwart, and A.J. van der Schaft, "Port representations of the transmission line," July 2005, IFAC World Congress 2005 (to appear).

[3] O.J. Staffans, "Passive and conservative continuous-time impedance and scattering systems. part I: Well-posed systems," Mathematics of Control, Signals, and Systems, vol. 15, pp. 291-315, 2002.

[4] R.F. Curtain and H.J. Zwart, An Introduction to Infinite-Dimensional Linear Systems Theory, Springer-Verlag, New York, 1995.

[5] Z.H. Luo, B.Z. Guo, and O. Morgul, Stability and Stabilization of Infinite Dimensional Systems with Applications, Springer-Verlag, 1999.

[6] E. Kreyszig, Introductory Functional Analysis with Applications, John Wiley \& Sons, New York, 1978.

[7] G. Tao and P. A. Ioannou, "Strictly positive real matrices and the Lefschetz-Kalman-Yakubovich lemma," IEEE Trans. on Automatic Control, vol. 33, no. 12, pp. 1183-1185, December 1988.

[8] J.A. Villegas, H. Zwart, and Y. Le Gorrec, "Boundary control systems and the system node," July 2005, IFAC World Congress 2005 (to appear).

[9] O.J. Staffans, Well-Posed Linear Systems, Cambridge University Press, 2005. 